\title{
A reflective temperature-insensitive all-fiber polarization-mode interferometer and pressure sensing application based on PM-PCF
}

\author{
Zhen Yin ${ }^{\mathrm{a}}$, Rong Gao ${ }^{\mathrm{b}}$, Youfu Geng ${ }^{\mathrm{c}}$, Xiaoling Tan ${ }^{\mathrm{d}}$, Xuejin $\mathrm{Li}^{\mathrm{e}}{ }^{\mathrm{e}}$ \\ ${ }^{a}$ College of Optoelectronic Engineering, Shenzhen Key Laboratory of Sensor Technology, Shenzhen \\ University, Shenzhen 518060, China. \\ ${ }^{\mathrm{b}}$ College of Electronic Science and Technology, Shenzhen University, Shenzhen 518060, China. \\ ${ }^{\mathrm{c} C}$ Collage of Physics and Energy, Shenzhen Key Laboratory of Sensor Technology, Shenzhen University, \\ Shenzhen 518060, China. \\ ${ }^{\mathrm{d}}$ The Electronic Communication Department, Shenzhen Institute of Information Technology, Shenzhen \\ 518172, China.
}

${ }^{\mathrm{e}}$ The Chinese University of Hong Kong (Shenzhen), Shenzhen 518172, China

Corresponding author: ${ }_{\text {lixuejin@ @zu.edu.cn }}$

\begin{abstract}
We experimentally demonstrate a novel reflective temperature-insensitive all-fiber pressure sensor based on polarization-maintaining photonic crystal fiber (PM-PCF). The sensing head is composed of a small segment of PM-PCF and a leading single-mode fiber (SMF). Two orthogonal polarized modes in the PM-PCF are excited by the splice point between SMF and the PM-PCF. After a round trip along the PM-PCF, a phase difference between two orthogonal polarization modes is induced due to the intrinsic high-birefringence of PM-PCF, and a Michelson-type reflective polarization-mode interferometer is enabled. The experimental results demonstrate that the wavelength-pressure coefficient of 4.15 $\mathrm{nm} / \mathrm{MPa}$ can be achieved, while the temperature coefficient is only $1.4 \mathrm{pm} /{ }^{\circ} \mathrm{C}$. This type of pressure sensor offers salient advantages of high wavelength-pressure coefficient, low cross sensitivity to temperature, compact size, robust structure, and flexibility in application.
\end{abstract}

Keywords: Polarization-maintaining photonic crystal fiber (PM-PCF), pressure sensor, birefringence.

\section{Introduction}

Fiber-optic pressure sensors are important in diverse applications, e.g., structural monitoring, petrochemical, aerospace etc [1-3]. In recent years, various pressure sensor based on photonic crystal fiber (PCF) have been extensively investigated for pressure measurements. Among them, the polarization-maintaining photonic crystal fiber (PM-PCF) has the optical property what the traditional polarization-maintaining fiber are not. It exhibits very low bending loss due to small-core diameter and the large numerical aperture. Moreover, the PM-PCF has the features of high birefringence, the flexible fabrication design, and reduced temperature sensitivity. Therefore, temperature-induced cross-sensitivity effects can be neglected for sensing applications, making it an attractive option for pressure sensing.

Nowadays, various configurations and fabrication techniques based on PCFs have been exploited to construct a pressure sensor. Pressure sensors using fiber Bragg grating (FBG) written in a PM-PCF [4] or index-guiding photonic crystal fiber [5] have been reported, but the fabrication technique for FBG is rather complex, which results in an expensive cost. Although a distributed fiber optic pressure sensor based on the all-solid photonic band gap fiber has been demonstrated [6], the sensor suffers 
cross-sensitivity with two pressure points, which could restrict the practical application as a distributed pressure sensor. Chen et al. proposed a pressure/temperature sensor with a dual-core photonic crystal fiber (DC-PCF) [7], however, its sensitivity of $-3.47 \mathrm{pm} / \mathrm{MPa}$ is relatively low. A PM-PCF based pressure sensor with polarimetric detection has also been demonstrated and proposed [8], but the polarimetric sensors are generally complex and not preferential in most applications. Wu et al. reported a PCF-based Fabry-Perot interferometer (FPI) [9] and its sensing capability for high-pressure and high-temperature measurements, but such a sensor is restricted with low pressure sensitivity and a high temperature cross-sensitivity. In addition, pressure sensors with PM-PCF based Sagnac interferometer have also been proposed and demonstrated [10-12]. However, this type of sensors has drawbacks of the complex installation technique, low efficiency of compatibility with another sensing head, and high loss on account of the employ of a 3-dB coupler.

In this paper, we propose a reflective temperature-insensitive all-fiber pressure sensor based on PM-PCF. Two orthogonal polarized modes are excited via splice point between leading single-mode fiber (SMF) and the PM-PCF. Then, the two orthogonal polarized modes transmit a round trip along the PM-PCF, and induce a phase difference due to the intrinsic high-birefringence of PM-PCF. So a smooth interference fringe is obtained as a result of Michelson-type reflective polarized modes interference. The experimental results demonstrate that the wavelength-pressure coefficient of 4.15 $\mathrm{nm} / \mathrm{MPa}$ can be achieved, and the temperature coefficient is only $1.4 \mathrm{pm} /{ }^{\circ} \mathrm{C}$. This type of pressure sensor has features of high wavelength-pressure coefficient, low cross sensitivity to temperature, compact size, robust structure, and flexibility in application.

\section{Experimental setup and principle}

Figure 1 illustrates the experimental setup of our proposed reflective temperature-insensitive all-fiber pressure sensor based on PM-PCF. The system is composed of a broadband source (BBS), a circulator, a single-mode fiber polarizer, a polarization controller (PC), an optical spectrum analyzer (OSA, Agilent 86146B) with a resolution of $0.06 \mathrm{~nm}$, and a PM-PCF served directly as pressure sensing element. The broadband source (BBS) is employed as an incident light source and the optical spectrum analyzer (OSA) is used to monitor the interference spectrum in real time. The PM-PCF we used is fabricated by the Yangtze Optical Fiber and Cable Company (YOFC Co., China). The PM-PCF is with a solid core surrounded by 6 rings of $2.2 \mu \mathrm{m}$ holes arranged in an 6-fold symmetry, the $\mathrm{d} / \Lambda<0.46$, where $\mathrm{d}$ is the hole diameter and $\Lambda$ is the pitch between holes. Two opposite holes of the first ring are substituted by $5.3 \mu \mathrm{m}$ holes in order to induce a symmetry break, causing the birefringence. The scanning electron micrograph (SEM) image of the transverse cross section of the PM-PCF is shown in the inset of Fig. 1. The far end of the PM-PCF is spliced with a short section (less than $1 \mathrm{~cm}$ ) of leading single-mode fiber (SMF) to protect the PM-PCF end face.

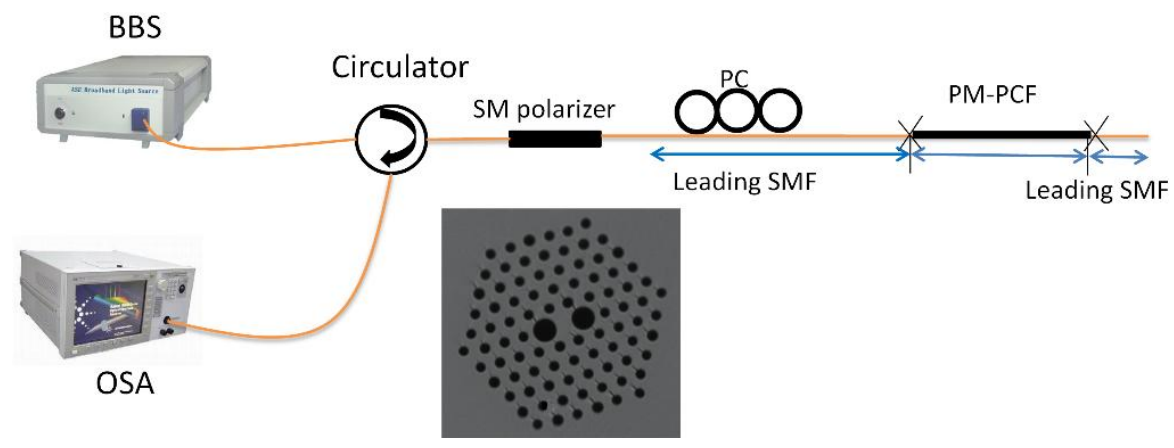


Fig. 1. Schematic diagram of the sensor setup. The inset is the scanning electron micrograph (SEM) image of the holes section of the PM-PCF.

As shown in Fig. 1, a circulator is used to lead the broad band source signal from the broadband source (BBS). The light becomes linearly polarized light after passing through the single-mode fiber polarizer; By adjusting the polarization controller, the linearly polarized light is launched at $45^{\circ}$ relatively to the eigen axes of PM-PCF, two orthogonal polarized modes are excited via the splice point between leading single-mode fiber and the PM-PCF. Then two orthogonal polarized modes are reflected at the end face of the leading SMF and recoupled back into the leading SMF again. A phase difference between two orthogonal polarization modes is induced due to the intrinsic high-birefringence of PM-PCF, and a Michelson-type reflective polarization-mode interferometer is enabled. So, a smooth interference fringe is obtained as a result of polarized modes interference.

The modal effective index and the birefringence of the PM-PCF are simulated based on a finite element method (FEM) with commercially available COMSOL software. The perpendicular wave module of the software is used to solve wave equations and to calculate the effective indices of the guided modes in the PM-PCF. Two polarized fundamental modes are found, corresponding to $\mathrm{LP}_{01-\mathrm{x}}$ and $\mathrm{LP}_{01-\mathrm{y}}$. From the simulating results, the phase birefringence $(\Delta \mathrm{n})$ is $5.85 \times 10^{-4}$ at the wavelength of $1550 \mathrm{~nm}$. Figures 2(a) and 2(b) illustrates the intensity profile of $\mathrm{LP}_{01-\mathrm{x}}$ and $\mathrm{LP}_{01-\mathrm{y}}$, and Fig. 2(c) and 2(d) are the corresponding energy contour maps, showing the energy is mainly limited in the core area of the PM-PCF.
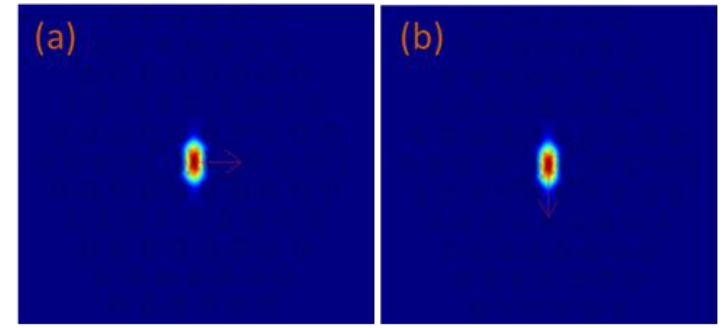

(c)

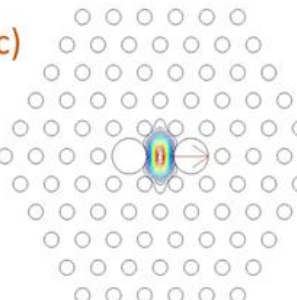

(d)

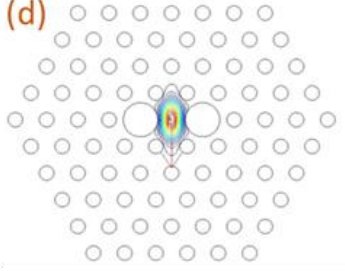

Fig. 2. The simulated mode fields (a, b) and energy contour map (c, d) of $\mathrm{LP}_{01-\mathrm{x}}$ and $\mathrm{LP}_{01-\mathrm{y}}$, respectively.

The output optical spectra are determined by the relative phase difference introduced to the two orthogonal polarized modes mainly by the PM-PCF. The total phase difference $\delta$ introduced by the PM -PCF can be expressed as

$$
\delta=\delta_{0}+\delta_{\mathrm{p}}
$$

where $\delta_{0}$ and $\delta_{\mathrm{p}}$ are the phase differences due to the intrinsic and pressure-induced birefringence over the length $2 L$ of the PM-PCF with,

$$
\delta_{0}=\frac{4 \pi B L}{\lambda}
$$




$$
\delta_{p}=\frac{4 \pi B_{p} L}{\lambda}=\frac{4 \pi\left(\mathrm{K}_{p} \Delta \mathrm{p}\right) L}{\lambda}
$$

Where, $B=n_{\mathrm{s}}-n_{\mathrm{f}}$ is the birefringence of the PM-PCF, $n_{\mathrm{s}}$ and $n_{\mathrm{f}}$ are the effective refractive indices along the slow and fast axis of the PM-PCF, respectively. $\lambda$ is the wavelength of the incident light. $\Delta p$ is the applied pressure, and $K_{\mathrm{p}}$ the birefringence-pressure coefficient of PM-PCF expressed as [13]

$$
K_{p}=\frac{\partial n_{s}}{\partial p}-\frac{\partial n_{\mathrm{f}}}{\partial p}
$$

The spacing $S$ between two adjacent fringes in the interference spectra can be approximated by

$$
S=\frac{\lambda^{2}}{2 B L}
$$

The pressure-induced wavelength shift of the transmission minima can be expressed as

$$
\Delta \lambda=\frac{S \cdot \delta_{p}}{4 \pi}
$$

Therefore, the relationship between wavelength shift and applied pressure can be simplified as

$$
\Delta \lambda=\left(\frac{K_{P} \cdot \lambda}{2 B}\right) \Delta p
$$

The above Eq. (7) clearly describes a linear relationship between the wavelength shift of the interference minima and the applied pressure variations. By measuring the shift value of the transmission dips by the optical spectrum analyzer (OSA), the applied pressure can be determined easily.

\section{Results and Discussions}

Experimentally measured reflection spectra at $1525-1610 \mathrm{~nm}$ with $L=6.3 \mathrm{~cm}, 9 \mathrm{~cm}$ and $12 \mathrm{~cm}$ are shown as Fig. 3. It is clearly seen that the interference signal contrast can be achieved as highly as 20 $\mathrm{dB}$ with an OSA resolution of $0.06 \mathrm{~nm}$. Also, it can be seen that the fringe spacing gets larger with fiber shortened. From Eq. (5), the intrinsic birefringence of the PM-PCF used in our experiment is $9.6 \times 10^{-4}$ at $1550 \mathrm{~nm}$.

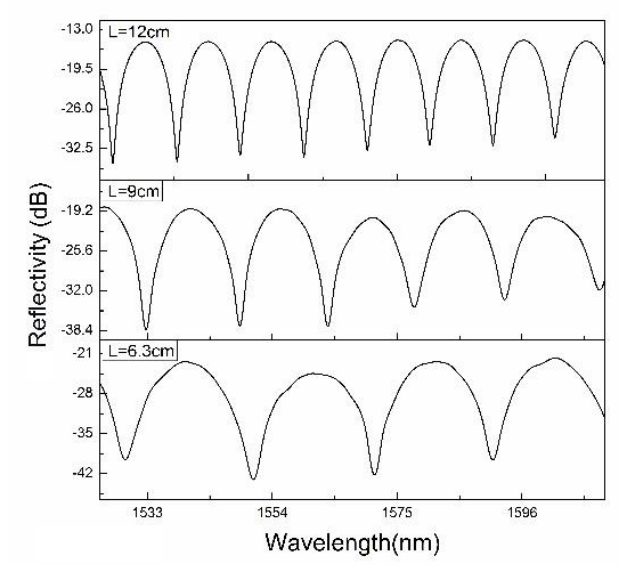

Fig. 3. Reflection spectra at $1525-1610 \mathrm{~nm}$ with $\mathrm{L}=6.3 \mathrm{~cm}, 9 \mathrm{~cm}$, and $12 \mathrm{~cm}$, respectively

In our experiment, the air pressure is initially at one atmospheric pressure (about $0.1 \mathrm{MPa}$ ); we can gradually increase air pressure from $0.1 \mathrm{MPa}$ to $1.2 \mathrm{MPa}$ with a step of $0.1 \mathrm{MPa}$ at room temperature. As shown in Fig. 4(a), when applied pressure increases, the spectrum shifts toward longer 
wavelengths. By measuring the wavelength shift of the transmission minima in the wavelength spectrum, the pressure variation can be determined. Figure 4(b) shows the experimental data of the wavelength shift versus applied pressure. It can be seen that there is a good linear relationship between the wavelength and applied pressure, and the correlation coefficient of $\mathrm{R}^{2}$ reaches 0.998 . Within the pressure range of 0.1-1.2 $\mathrm{MPa}$, the wavelength-pressure coefficients of the three pressure sensors can reach $4.06 \mathrm{~nm} / \mathrm{MPa}, 4.15 \mathrm{~nm} / \mathrm{MPa}$ and $4.1 \mathrm{~nm} / \mathrm{MPa}$ for PM-PCFs with lengths of $6.3 \mathrm{~cm}, 9 \mathrm{~cm}$ and 12 $\mathrm{cm}$, respectively. Therefore, the wavelength-pressure coefficient is independent of the length of the PM-PCF, as described in Eq. (6). So, in practical application, long PM-PCF or even coil the PM-PCF is not necessary as the other fiber Sagnac interferometers [10-12].
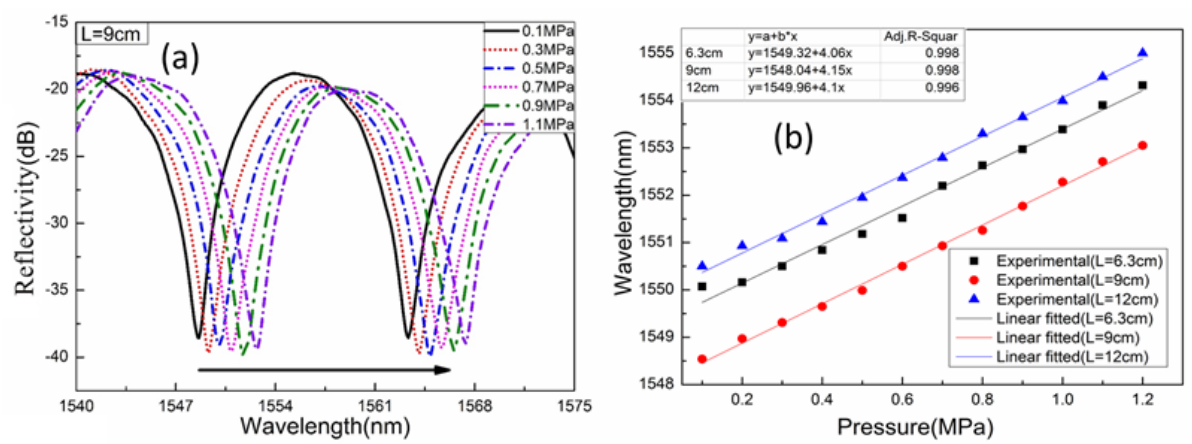

Fig. 4. (a) Reflection spectra of a $9 \mathrm{~cm}$ PM-PCF under different air pressures. (b) Wavelength shift versus applied air pressure with different lengths of PM-PCF.

Figure 5 illustrates the PM-PCF birefringence dependence on the air pressure with a PM-PCF length of $9 \mathrm{~cm}$. The sensitivity of the birefringence to the pressure can be calculated according to the Eq. (7) and is found to be approximately $5.15 \times 10^{-6} \mathrm{MPa}^{-1}$.

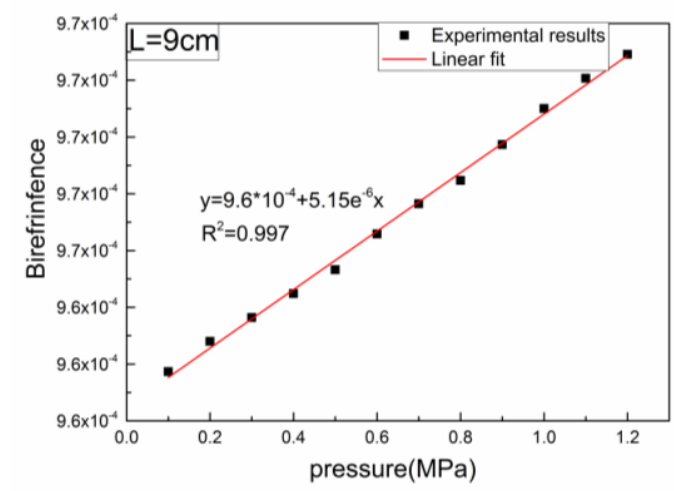

Fig. 5. Excess birefringence versus applied pressure.

Temperature sensitivity of the proposed sensor is also researched by placing the PM-PCF with length of $9 \mathrm{~cm}$ into an oven. We increase the range of temperature measurement from $25^{\circ} \mathrm{C}$ to $900{ }^{\circ} \mathrm{C}$. Figure 6 displays the wavelength shift of two transmission minima versus temperature with $R^{2}$ value of 0.9856 and 0.9956 , respectively. The measured temperature coefficient is about $1.4 \mathrm{pm} /{ }^{\circ} \mathrm{C}$, which is much smaller than $10 \mathrm{pm} /{ }^{\circ} \mathrm{C}$ of fiber Bragg grating. Based on theoretical analysis of the temperature dependence of the birefringence of the PM-PCF reported in [14], the temperature dependence of the birefringence of the PM-PCF is small. Since the core and the cladding resigns of the PM-PCF are composed of single material of silica with low thermo-optic and thermal expansion properties, the temperature coefficient is small and the sense head is insensitive to temperature. 


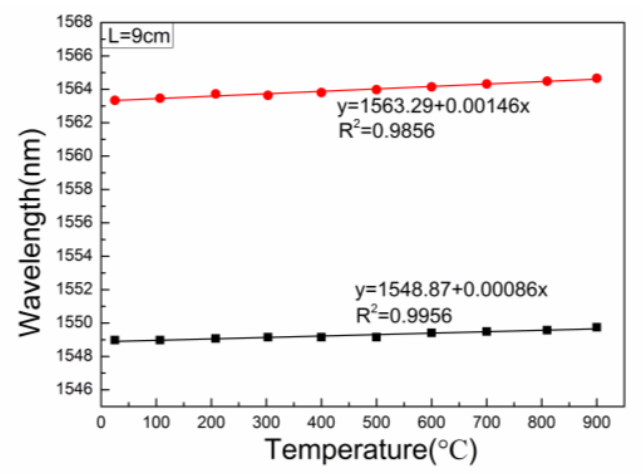

Fig. 6. Wavelength shift of the two transmission minimums against temperature.

Although the highest applied pressure is 1.2 MPa in our experiment, there is no obvious evidence showing any physical limitation by the PM-PCF itself. Therefore, higher pressure sensing is possible with this sensor. Compared with pressure sensor using a $79.6 \mathrm{~cm}$ long PM-PCF based Sagnac interferometer, the proposed sensor is more compact and flexible.

\section{Conclusion}

A reflective temperature-insensitive all-fiber pressure sensor based on PM-PCF is proposed and demonstrated experimentally. Experimental results and simplified theoretical analysis of the pressure sensor have been presented. The sensing head is composed of a small segment of PM-PCF and a leading single-mode fiber (SMF). A smooth interference fringe is obtained as a result of Michelson-type reflective polarized modes interference. The wavelength-pressure coefficient of 4.15 $\mathrm{nm} / \mathrm{MPa}$ can be achieved with an excellent linearity, while the temperature coefficient is only 1.4 $\mathrm{pm} /{ }^{\circ} \mathrm{C}$. This type of pressure sensor has features of high wavelength-pressure coefficient, low cross sensitivity to temperature, compact size, robust structure, and flexibility in application. Thus, the proposed pressure sensor is an ideal candidate for pressure sensing even in harsh environments.

\section{Acknowledgement}

This work is supported by the National Science Foundation of China (No. 61308046 and No. 61275125); The Research Foundation for the Doctoral Program of Higher Education of the Ministry of Education (20124408110003); The High-Level Talents Project of Guangdong Province and the Basic Research program of Shenzhen; Shenzhen Science and Technology Project; The High-Level Talents Project through the National Science Foundation, Shenzhen University (827.00002601).

\section{References}

[1] G. Y. Sun, D. S. Moon and Y. J. Chung, IEEE Photon. Technol. Lett. 19 (2007) 2027-2029.

[2] Y. Liu, B. Liu, X. H. Feng, W. G. Zhang, G. Zhou, S. Z. Yuan, G. Y. Kai and X. Y. Dong, Opt. 44 (2005) 2382-2390.

[3] Y. -G. Han, Appl. Phys. B 95 (2009) 383-387.

[4] C. Sonnenfeld and S. Eve, Sensors 11 (2011) 2566-2579.

[5] Z. Y. Liu, C. Wu, C. Lu and H. Y. Tam, Opt. Lett. 38 (2011) 1385-1387.

[6] W. H. Ding and Y. Jiang, Opt. Express 20 (2012) 14054-14063.

[7] D. R. Chen, G. F. Hu and L. X. Chen, Proc. SPIE 8307 (2011) 1N1-10.

[8] H. K. Gahir and D. Khanna, Appl. Opt. 46 (2007) 1184-1189.

[9] C. Wu, H. Y. Fu, K. K. Qureshi, B. O. Guan and H. Y. Tam, Opt. Lett. 36 (2011) 412-414.

[10] H. Y. Fu, H. Y. Tam, L. Y. Shao, X. Y. Dong, P. K. A. Wai, C. Lu and S. K. Khijwania, Appl. Opt. 47 (2008) 2835-2839. 
[11] H. Y. Tam, S. K. Khijwania and X. Y. Dong, 2007 AOE (2007) 345-347.

[12] H. Y. Fu, C. Wu, M. L. V. Tse, L. Zhang, H. Y. Tam, B. O. Guan and C. Lu, Appl. Opt. 49 (2010) 2639-2643.

[13] H. G. Thakur and S. Nalawade, Optics \& Laser Technl. 42 (2010) 1139-1144.

[14] D. H. Kim and J. U. Kang, Opt. Eng. 46 (2007) 75003/1-5. 